\title{
Controlled deposition of picoliter amounts of fluid using an ultrasonically driven micropipette
}

\author{
Bradley J. Larson, ${ }^{\text {a) }}$ Susan D. Gillmor, and Max G. Lagally \\ University of Wisconsin-Madison, Madison, Wisconsin 53706
}

(Received 30 May 2003; accepted 26 January 2004; published 8 March 2004)

\begin{abstract}
A fluid microplotter that uses ultrasonics to deposit small fluid features has been constructed. It consists of a dispenser, composed of a micropipette fastened to a piece of lead zirconate titanate piezoelectric, attached to a precision positioning system. When an electrical signal of the appropriate frequency and voltage is applied, solution in the tip of the micropipette wicks to the surface in a controlled fashion. The gentle pumping of fluid to the surface occurs when the micropipette is driven at frequencies in the range of $400-700 \mathrm{kHz}$. Spots with diameters smaller than several microns can be deposited in this manner. Continuous lines can also be produced. Several examples of deposited patterns and structures are described. This means of deposition represents a higher-resolution alternative to standard fluid deposition techniques in the fabrication of biological microarrays or polymer-based circuits. (c) 2004 American Institute of Physics.
\end{abstract}

[DOI: $10.1063 / 1.1688436]$

\section{INTRODUCTION}

The dispensing of picoliter volumes of fluids onto precise locations on a surface is becoming increasingly important in a variety of venues. Deposited features may need to be continuous (lines or arcs), discrete (spots), or even threedimensional (towers). A smaller size of these features is beneficial in a wide variety of applications, ranging from conductive-polymer-based electronic circuits to biological microarrays.

On one extreme, solution printing of polymer-based circuits shows potential as an inexpensive means of producing flexible electronics. Much effort has been directed toward adapting inkjet printers to deposit entire circuits. The end goal is to create a system in which circuit patterns could be defined in software and then rapidly printed onto a substrate. Inkjet printers have attracted interest for this application because they are inexpensive and deposit fluid rapidly. The production of microcircuits, however, requires the ability to draw continuous lines of a very small width. Inkjets, by their nature, cannot produce continuous lines but instead form lines from overlapping spots. They are currently limited to making features no smaller than $\sim 50 \mu \mathrm{m}$ wide. To overcome this limitation, a photolithographic patterning step has been required to form dense microcircuits, significantly limiting the ability to custom-design circuits. ${ }^{1}$

On the opposite extreme, the fabrication of dense biological microarrays relies on the ability to attach many different biological molecules to a surface in a small area. There are three principal processes used to create biological microarrays: Light-directed synthesis of short deoxyribonucleic acid (DNA) strands on a surface, chemical synthesis of DNA on a surface, and the spotting and attachment of prepared biomolecules to a surface.

\footnotetext{
a) Author to whom correspondence should be addressed; electronic mail: bjlarson2@students.wisc.edu
}

In the first technique, photolithographic methods familiar from the semiconductor industry are used to synthesize, base by base, short oligonucleotide strands with a specific sequence in each feature on a surface. ${ }^{2-4}$ This light-directed in situ DNA synthesis can produce tightly packed small features ( $\sim 20 \mu \mathrm{m}$ wide) but is limited just to the production of DNA microarrays. Although some work has been done in patterning short polypeptides in this manner, ${ }^{5}$ practical protein or other biomolecule-based microarrays may never be created in this fashion because of length limitations and protein folding problems.

A similar chemical-based in situ DNA synthesis technique uses inkjets to build short strands of DNA on a surface by selectively depositing the appropriate chemicals to build specific DNA sequences at specific spots. ${ }^{6,7}$ The custom creation of DNA microarrays using inkjets is simpler than lightdirected synthesis. Significantly less complex equipment is required as well. Arrays produced using inkjets are not as dense ( $\sim 200 \mu \mathrm{m}$ center-to-center spacing on spots) as those produced by the photolithographic techniques. Again, this approach is limited to only short strands of DNA.

The third technique differs from the other two in that previously prepared biological solutions are deposited. Finely machined quill pins are dipped in a biological solution and then tapped on a surface at a precise location to deposit a spot. ${ }^{8}$ If this surface is chemically activated in a particular way, the biomolecules in solution will bind to the surface within the spot. A microarray can thus be fabricated by spotting multiple different biological solutions across the surface. The greatest advantage of this technique is that it can be used with a wide variety of prepared biological solutions, including proteins and carbohydrates. ${ }^{9-11}$ These solutions can be very limited and expensive, so minimizing the use of solution per microarray produced is desirable. Existing quill pins for spotters are limited to producing spots roughly $75 \mu \mathrm{m}$ in 


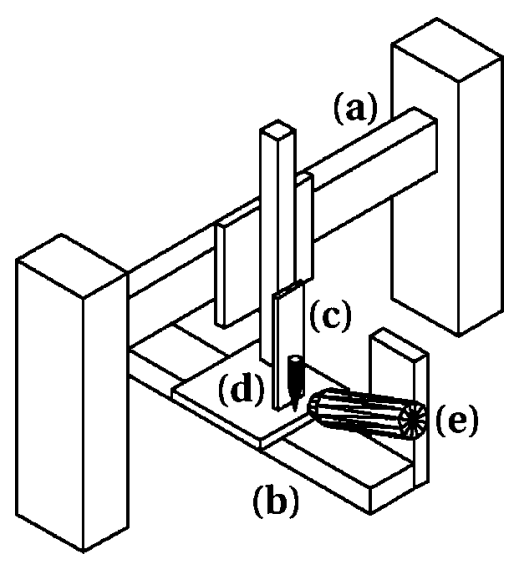

FIG. 1. A schematic diagram of the fluid microplotter. It is composed of a precision positioning system with (a) $X$, (b) $Y$, and (c) $Z$ positioning stages in the configuration shown and (d) a dispenser head composed of a piece of PZT piezoelectric attached to a micropipette, not shown to scale. The fluid deposition process is monitored by (e) a CCD camera.

diameter. On average, $1 \mathrm{~nL}$ of solution is deposited per spot using existing systems.

We have developed a fluid microplotter that can deposit discrete features that are an order of magnitude smaller than those listed above, using many times less solution. ${ }^{12}$ In addition, it can easily draw continuous lines and arcs as well as deposit high-aspect-ratio towers. The microplotter is able to handle a wide range of fluid viscosities. This article descibes the operation and use of this device.

\section{FLUID MICROPLOTTER DESIGN}

The microplotter consists of a dispenser that is positioned over a substrate using a high-precision positioning system. This positioning system is controlled by custom software on a desktop personal computer (PC) that permits complex synchronized movements and deposition patterns. The deposition process can be monitored in real time using an attached charge-coupled-device (CCD) camera. A schematic overview of the microplotter is shown in Fig. 1.

The dispenser head is composed of a micropipette attached to a piece of lead zirconate titanate (PZT) piezoelectric. When driven by an ac current, the PZT causes the micropipette to vibrate primarily along its axis. Under appropriate conditions (a driving frequency in the range 400-700 kHz, 1-4 V peak-to-peak driving voltage, tip inside diameters (IDs) ranging from $1 \mu \mathrm{m}-100 \mu \mathrm{m}$ ), applying the ultrasonic field causes fluid in the tip to wick out onto the surface. Smaller micropipette IDs yield smaller spots. For a given micropipette, the size of the resulting fluid feature can be controlled by raising or lowering the amplitude of the peak-to-peak ac voltage. As we will show later, the viscosity of the deposited solution also affects spot size.

This design is fundamentally different from that of inkjet printers or other piezoelectric-based fluid dispensers. In those designs, the expansion of a piezoelectric element exerts a force on fluid contained within a narrow channel, causing fluid to be ejected onto a surface. Other devices that use micropipettes use cylindrical piezoelectric elements surrounding the micropipette to squeeze fluid out of the mi- cropipette tip and thus operate on the same principle of a pressure pulse. ${ }^{13,14}$ Our dispenser is much simpler and the principle of operation is different, leading to much better performance. As will be described in more detail later, ultrasonic vibrations cause fluid to be ejected from the tip of the micropipette, not pressure due to the contraction of the capillary tube, as in these other designs.

To achieve the deposition of fluid features at precise locations, we use three positioning stages. Two stages move the dispenser head to give $X-Z$ positioning, while a third stage moves the substrate in $Y$. These stages can position the substrate and micropipette tip relative to each other over a 50 $\mathrm{cm} \times 50 \mathrm{~cm} \times 10 \mathrm{~cm}$ volume, with a rated maximum lateral positioning error of $20 \mu \mathrm{m}$. By tuning the proportionalintegral-derivative control loop used for positioning feedback and operating over a smaller portion of this volume (e.g., $5 \mathrm{~cm} \times 5 \mathrm{~cm} \times 2 \mathrm{~cm}$ ), we can achieve much more accurate lateral positioning (error of $<5 \mu \mathrm{m}$ ). A motor controller directs the motion of these stages and it, in turn, is controlled by software on a desktop PC. This software allows for completely automated deposition of complex patterns and shapes on a substrate using multiple feed fluids.

\section{DEPOSITION PROCESS}

The steps in depositing fluid onto a surface using the fluid microplotter are: Loading the dispenser with fluid, positioning the dispenser over the starting coordinate for the first feature, drawing the first feature, repeating until all features of the current fluid are drawn, discharging any remaining fluid, washing the dispenser tip, and if necessary, loading the dispenser with the next fluid.

The loading of the micropipette with fluid can be accomplished by either attaching a supply of fluid to the wide end of the micropipette or by dipping the sharp end of the micropipette into a fluid well. A continuous fluid supply is useful for a large amount of deposition using a single fluid, whereas dipping the micropipette tip into fluid is useful for depositing multiple fluids (dip-pen mode). We concentrated on the use of the device in a dip-pen mode, as the ability to take up very small fluid volumes is useful when expensive or limited-quantity fluids are used, something that is often the case in the fabrication of biological microarrays. When the sharp tip of the micropipette is placed into a fluid well, capillary action causes fluid to wick up into the tip. This process can be controlled so as to allow submicroliter control of the loaded fluid volume by controlling the time spent in the fluid well. A micropipette with a $1 \mu \mathrm{m}$ inside diameter at the tip can take up a dilute aqueous solution at a controlled rate of $\sim 5$ nanoliters per second.

To deposit a spot, the dispenser tip is lowered to the substrate at the desired $X, Y$ position, a pulse at the correct frequency and peak-to-peak voltage is sent to the PZT for a certain duration, and the dispenser tip is raised. The same frequency pulse is used for all solutions deposited using a particular dispenser. If the feature is continuous, such as a line or arc, the tip is lowered until the fluid makes contact with the substrate. A continuous ac signal is supplied, the tip is backed off the surface slightly while maintaining fluid 
contact, and the continuous feature is drawn by coordinating the movement of all three stages. The ac signal is then shut off and the tip is raised. Satellite droplets, a concern for inkjets, are not a problem for the microplotter because the only fluid deposited is from contact of the fluid at the dispenser tip with the surface. The gentle nature of the interaction of the micropipette tip with the surface means that thousands of spots can be deposited by a dispenser without degradation of its tip.

Accurate $Z$ positioning is important in order to guarantee the deposition of regularly sized and shaped fluid features. If the dispenser is positioned too high above the surface when attempting to deposit a feature, fluid contact may not be made and no feature will be deposited. Software that calculates the correct surface height at each $X, Y$ position is the primary means of precise $Z$-positioning control.

In the dip-pen mode, when all features for a particular solution have been drawn, the fluid remaining in the dispenser can be salvaged by returning the dispenser to rest over the original fluid well. An ac pulse of a higher peak-to-peak voltage than that used to deposit spots is sent to the PZT, causing the micropipette to spray any remaining solution back into the original well. Return of fluid is generally not necessary because of the precise loading characteristics of the device, which allow the fluid volume in the dispenser to be preadjusted to create the required number of features with no waste. The micropipette tip can be cleaned by loading a solvent, such as deionized water, and spraying it back out. This loading and spraying operation is repeated several times to reduce the possibility of cross contamination.

Clogging by dust and other particles is a general problem with micropipettes that have less than $5 \mu \mathrm{m}$ IDs at the tip. In our system, clogging is overcome by two means. We can unclog a tip simply by using a medium-voltage ac pulse to dislodge the clogging particles. When the micropipette tip is in a solvent well, often the uptake of solvent from the sharp end of the micropipette will force the clogging particle loose and carry it away from the tip. When combining these two methods, clogs can be quickly removed, allowing operation even with micropipette IDs smaller than $1 \mu \mathrm{m}$.

\section{PERFORMANCE TESTS}

We tested the capabilities of this system for smallvolume fluid deposition in several ways. Initial testing was carried out using aqueous solutions of food coloring to allow for easy analysis of fluid deposition runs using standard optical microscopes. Figure 2 shows the micropipette tip in the process of depositing a spot as well as a pattern made from two solutions with quite different viscosities, red and blue food coloring. The red food coloring viscosity is close to that of water, while the blue food coloring is significantly more viscous. To demonstrate the effect of viscosity on deposited spots, the applied frequency and voltage were held constant for the two solutions. Different spot sizes resulted $(28 \mu \mathrm{m}$ diameter for the red spots, $20 \mu \mathrm{m}$ diameter for the blue spots). Figure 3 shows that very small spots can be made,

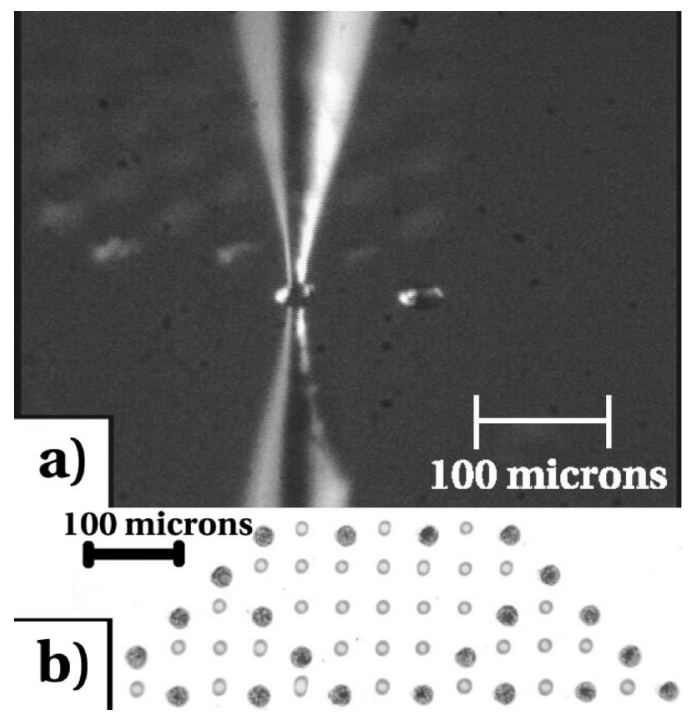

FIG. 2. Microplotter performance. (a) A close-up image of the micropipette tip in the process of depositing a pattern of spots. Several spots, spaced $\sim 100 \mu \mathrm{m}$ apart, are shown. (b) A pattern made from red and blue food coloring deposited on an untreated glass slide at the same dispenser voltage and frequency. The red spots are $28 \mu \mathrm{m}$ in diameter, the blue spots are 20 $\mu \mathrm{m}$ in diameter, and the center-to-center spacing of the spots is $50 \mu \mathrm{m}$. The spot size differences are caused by the differing viscosities of the two solutions.

even with dilute aqueous solutions on a hydrophilic substrate. In both of these tests, the glass slides were not treated or cleaned.

For biological applications, small spots are desired because of the significantly lower volumes of solution deposited. To estimate how much solution is used per spot deposited by the microplotter, the dispenser head was loaded with a known volume of solution. The microplotter then was commanded to deposit as many spots as it could before it ran out of solution. By counting the resulting number of spots, it was determined that the microplotter uses approximately $16 \mathrm{pL}$ per spot for $50 \mu \mathrm{m}$ diameter spots and $3 \mathrm{pL}$ per spot for 20 $\mu \mathrm{m}$ diameter spots. These values are lower than what is expected by modeling each spot as a hemisphere, but, as can be seen in Fig. 2(a), the deposited spots are not full hemispheres, so these values are reasonable.

The ability to draw continuous features, such as lines or arcs, is a unique capability of the microplotter. To demon-

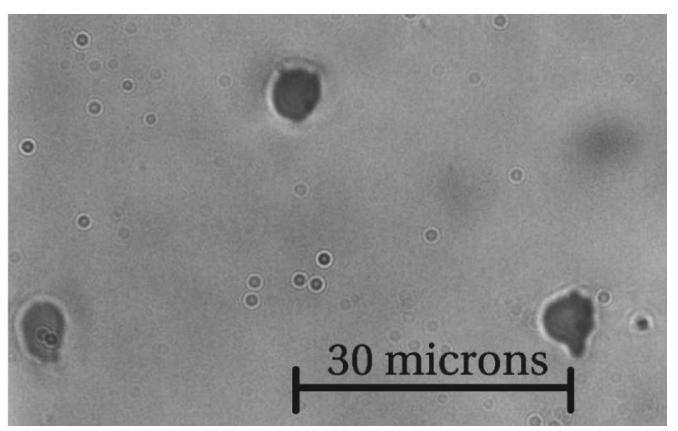

FIG. 3. An example of small feature sizes: A triangular pattern of $6 \mu \mathrm{m}$ diameter spots in red food coloring on an untreated glass slide. Even dilute low-viscosity aqueous solutions deposited on a hydrophilic substrate can be controlled to give uniform spots of a small size. 


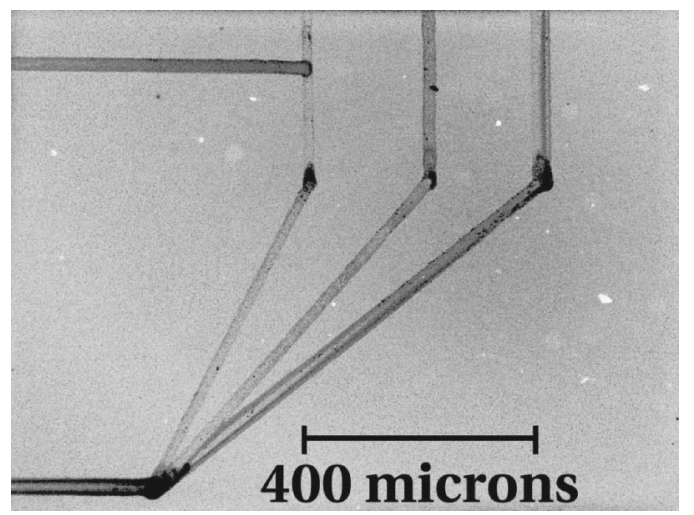

FIG. 4. An example of continuous-feature deposition. A pattern of lines was deposited in blue food coloring onto an untreated glass slide. The lines are approximately $25 \mu \mathrm{m}$ wide.

strate this capability, a computer-aided design file containing a pattern was loaded into the controlling software of the device and the pattern was then deposited on a glass slide using blue food coloring. A result is shown in Fig. 4.

We have also tested this device with other solutions. One such test involved depositing DNA onto a glass slide to demonstrate the ability to fabricate biological microarrays. A solution containing double-stranded salmon sperm DNA at a concentration of $500 \mu \mathrm{g} / \mathrm{mL}$ was loaded into the dispenser and deposited in a pattern on a poly-l-lysine-coated slide. That slide was then washed with deionized water and ethanol, stained with an intercalating dye that fluoresces at 488 $\mathrm{nm}$, and washed again. The slide was scanned using a commercial microarray scanner. Figure 5(a) shows that the DNA was electrostatically attached to the surface in the specified pattern. The size of the deposited spots was measured in a scanning laser confocal microscope. The spots, shown in Fig. 5 (b), are $\sim 25 \mu \mathrm{m}$ in diameter. The fluorescent specks in both images away from the pattern are not due to errant droplets from the plotter, but are believed to be due to dust particles on the slide surface.

Earlier, we showed continuous lines deposited in food coloring. As a demonstration of the small line widths the microplotter can draw, a dilute gold nanoparticle solution was deposited on a silicon wafer, yielding lines less than 5 $\mu \mathrm{m}$ wide. Figure 6 shows that these lines are long and con-

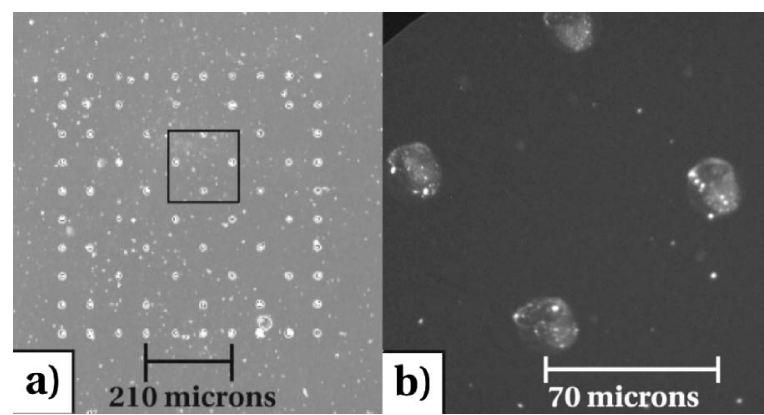

FIG. 5. Image of a pattern deposited using a $500 \mu \mathrm{g} / \mathrm{mL}$ solution of doublestranded DNA, which was then fluorescently stained with an intercalating nucleic-acid dye. The specks of fluorescence surrounding the pattern are due to dust on the glass surface. (a) The overall pattern was scanned using a commercial microarray scanner. (b) To examine the spots, a laser confocal microscope was used. The spots are $\sim 25 \mu \mathrm{m}$ in diameter.

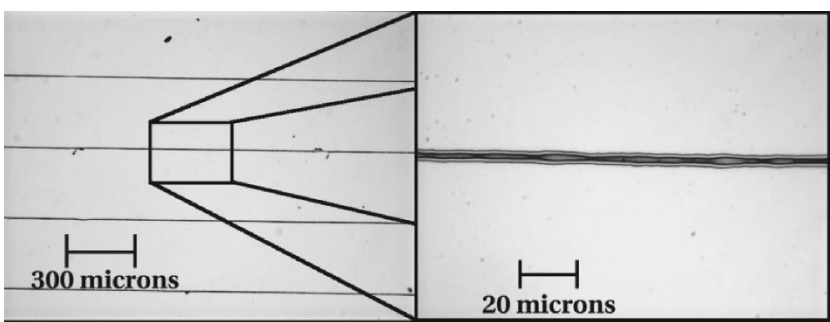

FIG. 6. Lines of a dilute gold nanoparticle solution deposited on a silicon wafer. The lines are $5 \mu \mathrm{m}$ wide and are continuous, except where broken by large particles present on the wafer.

tinuous, broken only where large particles were present (the wafer was not cleaned before fluid deposition).

Three-dimensional features can also be drawn using the microplotter, another unique capability. Figure 7 shows an example, a tower $\sim 30 \mu \mathrm{m}$ wide and $\sim 370 \mu \mathrm{m}$ tall deposited using a colloidal silver suspension. To create the tower, the micropipette, loaded with colloidal silver suspended in methanol, was brought in contact with the substrate (a silicon wafer) and ultrasonic vibrations were initiated. As the methanol evaporated at the micropipette tip, the suspended silver concentrated near the surface. By gradually drawing the dispenser away from the surface, silver was deposited in the shape of a tower.

\section{DISCUSSION}

The process by which longitudinal ultrasonic vibrations cause fluid to wick out of a micropipette tip in a controllable manner is not understood. Ultrasonics has been used in travelling-flexural-wave pumps for microfluidic systems. ${ }^{15}$ Additionally, ultrasonically vibrating micromachined silicon needles have been explored for possible use as pumps. ${ }^{16}$ These pump designs are more complex than our micropipette-based system and operate at different conditions. The fluid spraying mode of our micropipette tip appears to be similar to that reported previously, ${ }^{16,17}$ but there have been no reports of controlled fluid ejection onto a surface as we show here and it seems unlikely that the same mechanisms are at play.

An apparent change in wetting of fluid on the micropipette occurs when it is ultrasonically driven. Fluid rises up the outside walls of the micropipette when it is placed in a fluid well and made to resonate. Figure 8 shows the difference in wetting behavior for a resting and an ultrasonically driven micropipette. The micropipette tip was intentionally

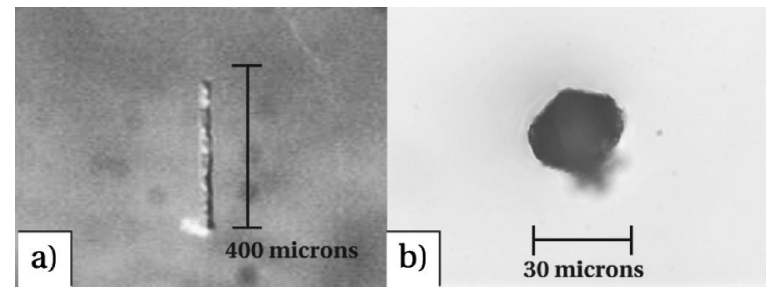

FIG. 7. A tower of silver particles deposited from a methanol-based colloidal silver suspension. (a) The height of the tower, $\sim 370 \mu \mathrm{m}$, can be seen in an image captured using the microplotter's attached CCD camera. (b) A top view image of the tower was taken using a standard optical microscope to determine its width $(\sim 30 \mu \mathrm{m})$. 


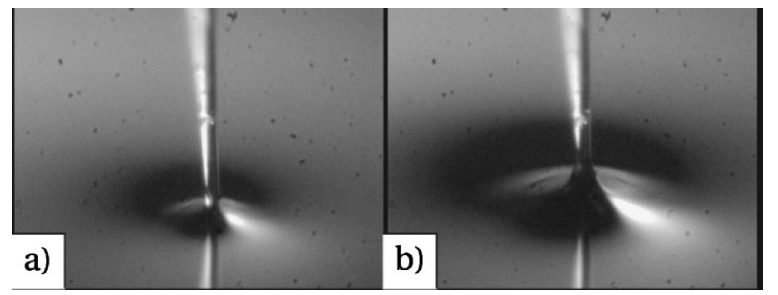

FIG. 8. Apparent change in wetting behavior caused by an applied ultrasonic pulse. We placed a glass micropipette with a sealed tip in water and compared wetting behavior with (a) no signal on the piezoelectric and (b) a signal at the resonant frequency of the dispenser. At the resonant frequency, water rises up the side of the glass micropipette.

sealed to demonstrate that this phenomenon is not caused by pumping from within the micropipette. Because we currently cannot predict the resonant frequency of a given dispenser, this change in wetting behavior is used to determine the resonant condition.

Several factors influence the sizes of fluid features produced by the fluid microplotter. The first is the size of the opening at the tip of the dispenser. The images we show illustrate fluid features produced by a variety of tip inside diameters. The smallest features (Fig. 3) were produced using an inside diameter of $1 \mu \mathrm{m}$, smaller than those of dispensers used for the other images. Second, fluid viscosity affects feature size. The more viscous the fluid, the less likely it is to spread from the dispenser tip. Third, the interaction of the surface with dispensed fluid affects feature size. If an aqueous solution is being dispensed onto a hydrophobic substrate, feature size is reduced. By adjusting these factors, it should be possible to create features smaller than $5 \mu \mathrm{m}$ wide.

\section{ACKNOWLEDGMENTS}

Funding for this research was provided by the University of Wisconsin-Madison's Office of University-Industry Relations. The authors thank Professor Dan van der Weide for the use of his confocal microscope.

${ }^{1}$ H. Sirringhaus, T. Kawase, R. H. Friend, T. Shimoda, M. Inbasekaran, W. Wu, and E. P. Woo, Science 290, 2123 (2000).

${ }^{2}$ S. P. A. Fodor, J. L. Read, M. C. Pirrung, L. Stryer, L. A. Tsai, and D. Solas, Science 251, 767 (1991).

${ }^{3}$ M. Chee et al., Science 274, 610 (1996).

${ }^{4}$ S. Singh-Gasson, R.D. Green, Y. Yue, C. Nelson, F. Blattner, M. R. Sussman, and F. Cerrina, Nature Biotechnol. 17, 974 (1999).

${ }^{5}$ K. N. Lee, D. S. Shin, Y. S. Lee, and Y. K. Kim, J. Micromech. Microeng. 13, 18 (2003).

${ }^{6}$ A. P. Blanchard, R. J. Kaiser, and L. E. Hood, Biosens. Bioelectron. 11, 687 (1996).

${ }^{7}$ T. R. Hughes et al., Nature Biotechnol. 19, 342 (2001).

${ }^{8}$ M. Schena, D. Shalon, R. Davis, and P. Brown, Science 270, 467 (1995).

${ }^{9}$ H. Zhu et al., Science 293, 2101 (2001).

${ }^{10}$ G. MacBeth and S. L. Schreiber, Science 289, 1760 (2000).

${ }^{11}$ D. Wang, S. Liu, B. J. Trummer, C. Deng, and A. Wang, Nature Biotechnol. 20, 275 (2002).

${ }^{12}$ B. J. Larson, C. H. Lee, A. Lal, and M. G. Lagally, U.S. Patent pending.

${ }^{13}$ R. C. Gamble, T. P. Theriault, and J. Baldeschwieler, US Patent No. 6,001,309 (December 14, 1999).

${ }^{14}$ G. C. Sasaki, U.S. Patent No. 6,296,811 (October 2, 2001).

${ }^{15}$ R. M. Moroney, R. M. White, and R. T. Howe, Proc. Transducers 1991, 931 (1991).

${ }^{16}$ A. Lal, Ph.D. thesis, University of California, Berkeley, 1996.

${ }^{17}$ M. Kurosawa, T. Watanabe, A. Futami, and T. Higuchi, Sens. Actuators A 50, 69 (1995). 